\title{
The Influence on the Walking Ability by the Different Load Support with a Robot Suit for Clinical Application of Robot Therapy
}

\author{
Toshiaki Tanaka, Ryo Matsumura, Norio Kato, Nobuya Hashimoto
}

\begin{abstract}
Purpose: The use of robots in rehabilitation is a relatively recent development and a growing area that is rapidly making inroads in clinical practice. This research examined changes in parameters when a gait-assist robot was worn and the use of a kinematic measurement method to examine the effects of differences in support and loading resulting from the use of a gait-assist robot on gait. Methods: The measured task was a walking task performed on a treadmill equipped with a robot suit. The measurement involved recording images with four digital video cameras, with one trial measured under each condition and a rest period between trials. The treadmill speed was set to $1.5 \mathrm{~km} / \mathrm{h}$, and steady-state gait was measured with a robot suit mounted over the treadmill. A motion analysis system was used for the analysis. The sampling rate was $60 \mathrm{~Hz}$. Three-dimensional motion analysis was used to calculate joint angles (degrees) during the walking task. The joint angles and the robot suit and the body joint angles were then compared under two conditions, the condition 1 (Assist 1) and the condition 2 (Assist 3). Each condition was performed five times (five gait cycles). Results: according to the comparison of joint angles under the condition of Assist 1 and Assist 3, the mean peak flexion of hip, knee, and mean peak dorsiflexion of ankle joints of assist 3 was greater than that of assist 1 during the swing phase of one gait cycle. For the comparison of HAL and the body joint angles, the misalignment may arise between the assist robot (robot suit) and the body during the period of swing phase of one gait cycle walking as a characteristic of exoskeleton-type gait-assist robots. The value $(\mathrm{mm})$ of the knee joint of misalignment between the robot suit and body of Assist 1 was significantly greater than that of Assis 3. Conclusion: The misalignment that may arise between the assist robot (robot suit) and the body during walking will need to be investigated in detail. Robot therapy should be used by understanding the characteristic of assist level of an exoskeleton-type gait-assist robot for effective gait training of versatile patients.
\end{abstract}

Index Terms - robot therapy, robot suit, assist level, gait training, motion analysis.

Toshiaki Tanaka, Faculty of Health Sciences, Hokkaido University of Science, Sapporo, Japan.

The Research Center for Advanced Science and Technology / Institute of Gerontology, The University of Tokyo, Tokyo, Japan

Ryo Matsumura, Faculty of Health Sciences, Hokkaido University of Science, Sapporo, Japan.

Norio Kato, Faculty of Health Sciences, Hokkaido University of Science, Sapporo, Japan.

Nobuya Hashimoto, Faculty of Health Sciences, Hokkaido University of Science, Sapporo, Japan.

\section{INTRODUCTION}

The use of robots in rehabilitation (robotic rehabilitation) is a relatively recent development and a growing area that is rapidly making inroads in clinical practice. The first robotic rehabilitation system was based on the concept of continuous passive motion $[1,2]$. The first exoskeleton-type powered suit for therapeutic use was introduced in the 1970s for patients with spinal cord injury [3]. Since then, robots have frequently been used in rehabilitation. A variety of support robots that reflect the application of engineering technology have been developed and used widely in the field of rehabilitation in recent years to bring about a rich, long-lived society that supports the independence of the aged and those with disabilities.

Research on the application of robots in rehabilitation (hereafter referred to as "robotic rehab") has given rise to expectations in the fields of medicine and social welfare in recent years, and clinical research in this area has been gradually expanding in Japan. One type that has drawn attention involves robots that are fitted directly to the human body to provide a power-assist function. Studies have been conducted on the use of power-assist robots for the support of long-term care, rehabilitation, and daily activities.

Robotic rehab for post-stroke gait disturbance, which mainly involves externally supporting the movement of the hip and knee joints of paralyzed lower extremities, has expanded. In rehabilitation training, robots have been used to provide patients with external assistance for optimal strength (the minimum assistance needed to accomplish a task) and sufficient precise repetition for motor learning, which is anticipated to improve the efficiency of rehabilitation training. Examples of training support robots are Lokomat(C) from Hocoma and Gait Trainer@ from Reha-Stim Medtec [4, 5]. The main advantages at present of introducing robots are that they can be used for training in repetitive movements, which are difficult for patients, and as a basis for enabling PTs to concentrate on qualitative training, As a clinical application to rehabilitation, robot therapy is actively researching from the viewpoint of motor learning [6, 7].

It was reported that independent walking was significantly increased by adding training using support robots in addition to normal training [8]. However, there are few studies that simultaneously verify a gait analysis on the robot side and the human side while wearing a walking support robot, and also the effect of support using a walking support robot is not clear. 


\section{The Influence on the Walking Ability by the Different Load Support with a Robot Suit for Clinical Application of Robot Therapy}

This research examined changes in parameters when a gait-assist robot was worn and the use of a kinematic measurement method to examine the effects of differences in support and loading resulting from the use of a gait-assist robot on gait.

\section{MATHODS}

\section{A. Subjects}

The subjects were four healthy people. The selection standards for the participants were those who had not fallen over during the previous year, those who had not suffered serious pain and musculoskeletal or neurological damage, those who were not on medication that might impair balance. The protocol for this study was approved by the ethics committee of the University of Tokyo (No.20-210).

\section{B. The specification of the gait-assist robot}

The Robot Suit HAL (hereafter referred to as "HAL") exoskeleton-type gait-assist robot, developed by University of Tsukuba, was used in this study. HAL is equipped with actuators for the hip and knee joints and specialized shoes with reaction force plate sensors. The mode and assist settings for HAL are indicated below:

(1)Voluntary control mode: Myoelectric potentials (motor units) are sensed from electrodes for the flexor and extensor muscles of the hip and knee joint, and the center of foot pressure is sensed from specialized shoes. An assist level is then selected, and joint movement is controlled at the calculated "assist torque (Nm)."

(2) Impedance control mode: Weight-bearing and joint movement are smoothly controlled (synonymous with voluntary control mode without assist).

(3)Assist level: The settings for the hip and knee joint actuators can each be adjusted to a level of 0 to 20 .

[assist level $\times$ myoelectric potential $=$ assist torque (maximum actuator output of $42 \mathrm{Nm}$ )]

\section{Measurement and Analysis}

The measured task was a walking task performed on a treadmill equipped with Robot Suit HAL. The measurement involved recording images with four digital video cameras, with one trial measured under each condition and a rest period between trials. The treadmill speed was set to 1.5 $\mathrm{km} / \mathrm{h}$, and steady-state gait was measured with Robot Suit HAL mounted over the treadmill.

Table 1 showed the walking conditions with HAL. Furthermore, table indicated the definition of joint angle by use of HAL in Table 2.

The Frame-DIAS (DKH Co,.Ltd.) motion analysis system was used for the analysis. The sampling rate was $60 \mathrm{~Hz}$. Three-dimensional motion analysis was used to calculate joint angles (degrees) during the walking task. The joint angles and the HAL and the body joint angles were then compared under two conditions, the conditon1 (Assist 1) and the conditon2 (Assist 3).
Table 1. The condition setting for HAL

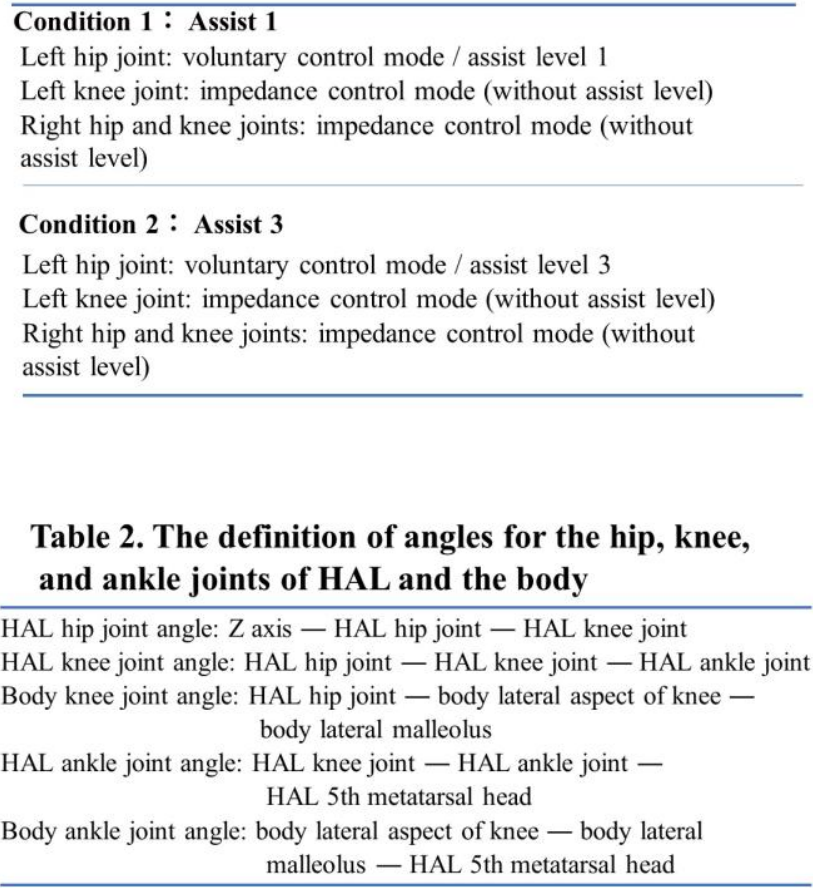

Each condition was performed five times (five gait cycles). Moreover, since the hip joint and foot of the body were surrounded by the robot suit, these joint motions could not measure, so only the knee joint and ankle joint were measured.

\section{RESULTS}

As a result, 4 subjects showed the same tendency. Therefore, these figures showed the data of one representative example.

\section{A. Comparison of joint angles under the Assist 1 and Assist 3 conditions in Fig. 1-3.}

With respect to joint angles, the maximal joint angle in the swing phase was $29.8^{\circ}$ under Assist 1 and 35.9 under Assist 3.

With respect to the time factor, the stance phase time was 1.98 seconds under Assist 1 and 1.73 seconds under Assist 3.

Mean peak of hip joint of assist 3 was greater than that of assist 1 during the swing phase of one gait cycle [9].

The maximal flexion angle of the knee joint during the swing phase was 53.9 under Assist 1 and 63.8 under Assist 3. Mean peak flexion of knee joint of assist 3 was greater than that of assist 1 during the swing phase of one gait cycle. The maximal dorsiflexion angle during the swing phase was 5.0 under Assist 1 and $13.9^{\circ}$ under Assist 3. Mean peak dorsiflexion of ankle joint of assist 3 was greater than that of assist 1 during the swing phase of one gait cycle. 


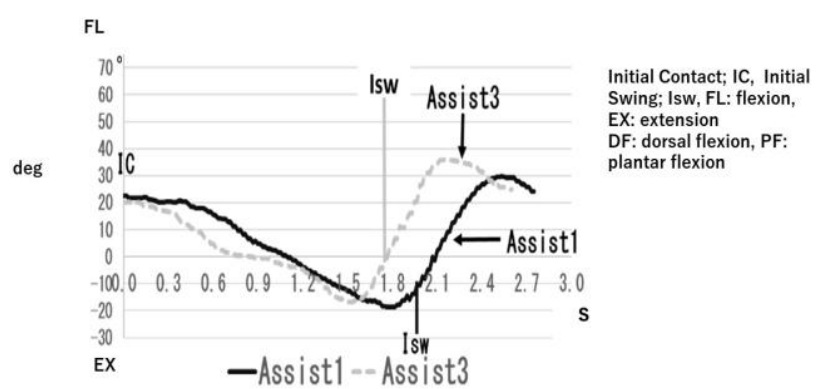

Fig 1 . Angle change in a single gait cycle of hip joint with use of HAL with comparing to Assist 1 and Assist 3 of an assist level.

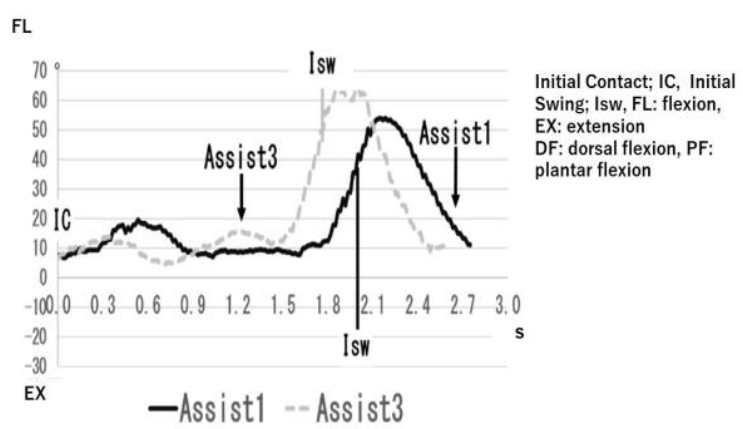

Fig 2. Angle change in a single gait cycle of knee joint with use of HAL with comparing to Assist 1 and Assist 3 of an assist level.

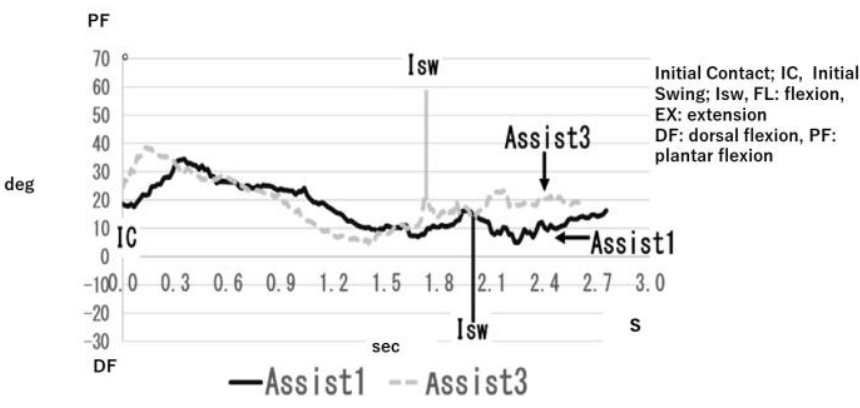

Fig 3. Angle change in a single gait cycle of knee joint with use of HAL with comparing to Assist 1 and Assist 3 of an assist level.

\section{B. Comparison of HAL and the body joint angles in Fig.}

4-7 and Table 3.

The misalignment may arise between the assist robot (Robot Suit HAL) and the body during the period of swing phase of one gait cycle walking as a characteristic of exoskeleton-type gait-assist robots (Table 3$)$. The value ( $\mathrm{mm}$ ) of the knee joint of misalignment between HAL and body of Assist 1 was significantly greater than that of Assis 3. In addition, especially, the direction of ankle joint movement during the swing phase was plantar flexion for HAL, while the direction of joint movement for the body was dorsiflexion.

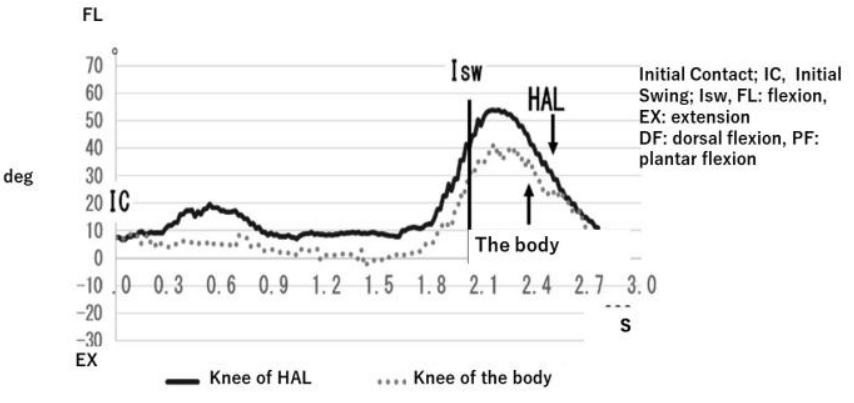

Fig 4. Angle change in a single gait cycle of knee joint of the body and HAL in the condition of Assist 1 of an assist level.

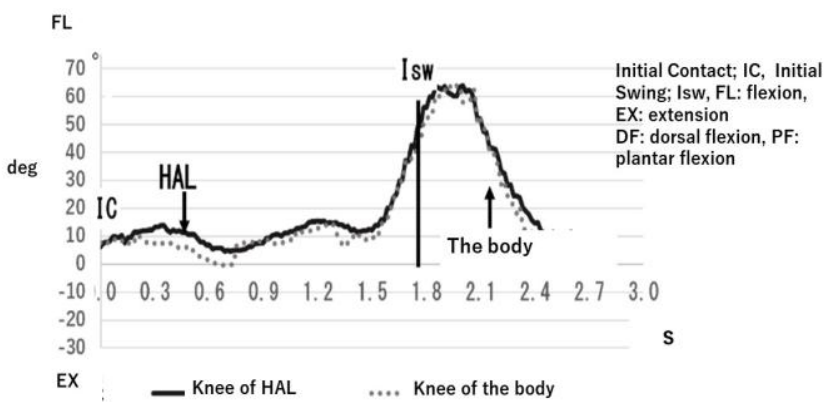

Fig 5. Angle change in a single gait cycle of knee joint of the body and HAL in the condition of Assist 3 of an assist level.

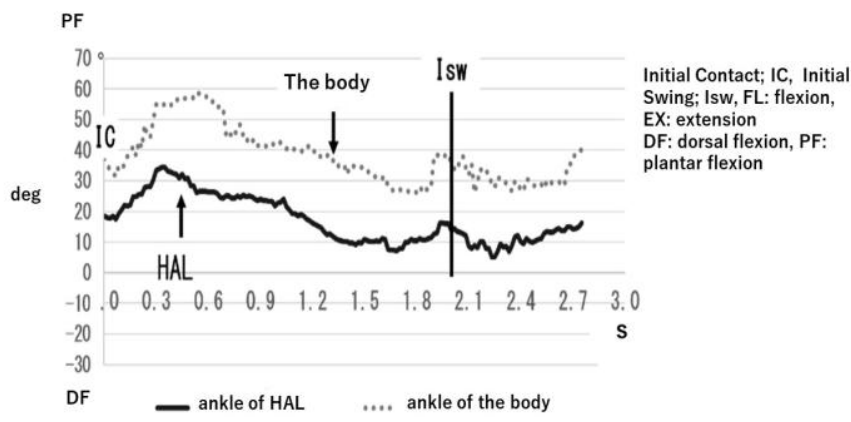

Fig 6 . Angle change in a single gait cycle of ankle joint of the body and HAL in the condition of Assist 1 of an assist level.

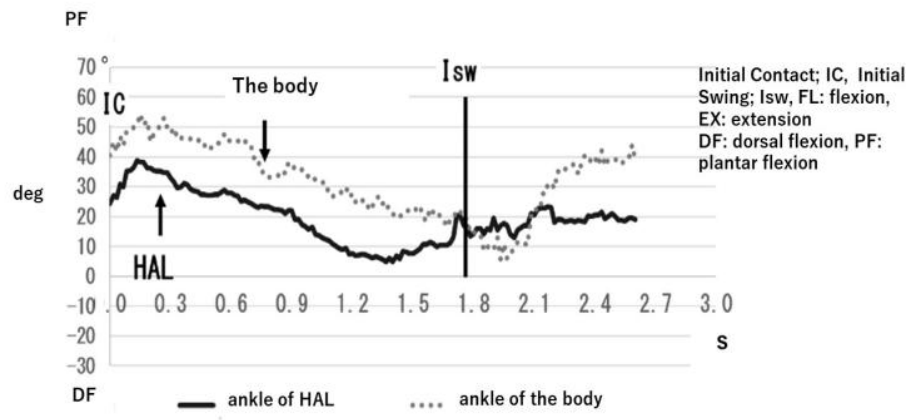

Fig 7. Angle change in a single gait cycle of ankle joint of the body and HAL in the condition of Assist 3 of an assist level. 
Table 3. The misalignment between the assist robot (Robot Suit HAL) and the body during the period of swing phase of walking (a single gait cycle).

Condition 1 (Assist 1) Condition 2(Asist 3)

\begin{tabular}{|c|c|c|c|}
\hline $\begin{array}{l}\text { The value }(\mathrm{mm}) \text { of misalignment } \\
\text { of the knee joint of between hal } \\
\text { and body }\end{array}$ & $12.4 \pm 4.7$ & $6.25 \pm 3.2$ & * \\
\hline $\begin{array}{l}\text { The value }(\mathrm{mm}) \text { of misalignment } \\
\text { of the ankle joint between hal and } \\
\text { body }\end{array}$ & $6.0 \pm 3.6$ & $3.2 \pm 2.6$ & \\
\hline
\end{tabular}

\section{DISCUSSION}

\section{A. Comparison of Assist 1 and Assist 3}

With regard to the hip joint angle, increasing the assist torque for hip joint extension during the stance phase by increasing the assist level from 1 to 3 resulted in a reduction in stance phase time. Increasing the assist torque for hip joint flexion during the swing phase resulted in an increase in the maximal flexion angle. With regard to the knee joint angle, an increase in the maximal angle of knee joint flexion was seen during the swing phase. Inertial force resulting from the increase in the assist torque for swing-phase hip joint flexion may have affected knee joint movement in impedance control mode. With regard to the ankle joint angle, a trend toward a smaller maximal dorsiflexion angle in the swing phase was seen in the Assist 3. Because there is no actuator control of the ankle joint, the ankle joint may move to plantar flexion naturally at around the Initial swing (hereinafter referred to as Isw ) [9] .

\section{B. Comparison of HAL and the body}

The misalignment $(\mathrm{mm})$ may arise between the assist robot (Robot Suit HAL) and the body during the period of swing phase of walking. The value of the knee joint of misalignment between HAL and body of Assist 1 was significantly greater than that of Assis 3. Because the assist level of Assist 1 is weaker than Assist 3, so the control of the joint movement of the body may be small in the condition of Assist 1 . Especially, a comparison of HAL and the body indicated that large misalignment occurred when differences were seen in plantar flexion and dorsiflexion, based on the shape of the graph for a single gait cycle. Under the Assist 3 condition, the ankle joint showed differences in movement direction for HAL and the body from the terminal stance to the Isw of the body, accompanied by the elimination of plantar flexion of the body ankle joint. The misalignment between HAL and the body with movement of the body ankle joint may have resulted from changes in hip and knee joint movement caused by differences in the hip joint assist torque.

Since 2020, an exercise therapy dimension has been added for single-joint HAL, and experience and knowledge involved in robot therapy in fields such rehabilitation and training have also been incorporated into physical therapy. In addition, investigations of the effectiveness of this approach are being aggressively pursued. As devices for assisting people in walking independently, however, they have one major problem. The problem is in the realm of the robot technology. Because the robots assist the movement of joints-primarily the hip and knee joints- along a single axis, they cannot prevent problems such as sudden falls. Consequently, they are strictly for assisting walking on level ground, they require monitoring, and their use is limited to rehabilitation programs that also use equipment to prevent falls. Robot models for assisting multi-joint, multiaxial movement of the lower extremity joints are at the research and development stage at organizations such as universities and companies.

\section{CONCLUSION}

This study obtained measurement data for small subjects. In near future, it will therefore be necessary to increase the amount of data by, for example, increasing the numbers of participants and trials and conducting statistical analyses. In addition, misalignment that may arise between the assist robot and the body during walking as a characteristic of exoskeleton-type gait-assist robots will need to be investigated in detail. Robot therapy should be used by understanding the characteristic of assist level of an exoskeleton-type gait-assist robot for effective gait training of versatile patients.

\section{ACKNOWLEDGMENT}

The authors would like to thank all subjects who kindly participated in the study.

\section{FUNDING}

This work was supported by Northern Advancement Center for Science and Technology and JSPS KAKENHI Grant Number JP. 20K20494.

\section{REFERENCES}

[1] D Khalili, M Zomlefer. An Intelligent robotic system for rehabilitation of joints and estimation of body segment petameters. IEEE Trans Biomed Eng.35(2):138-146, 1988.

[2] P.S. Lum, D.J. Reinkensmeyer, S.L. Lehman, "Robotic assist devices for bimanual physical therapy: Preliminary experiments", IEEE Transactions on Rehabilitation Engineering. 1(3):185-191, 1993.

[3] M. Vukobratovic, D. Hristic \& Z. Stojiljkovic. Development of active anthropomorphic exoskeletons. Med. Biol. ENG. 12(1):66-80, 1974.

[4] M Wirz 1, DH Zemon, R Rupp, A Scheel, G Colombo, V Dietz, TG Hornby. Effectiveness of automated locomotor training in patients with chronic incomplete spinal cord injury: a multicenter trial. Arch. Phys. Med. Rehabil. 86:672-680, 2005.

[5] S Hesse and D Uhlenbrock. A mechanized gait trainer for restoration of gait. J Rehabil Res Dev. 37(6): 701-708,2000.

[6] A Duschau-Wicke, J V Zitzewitz, A Caprez, L Lunenburger, R Riener.Path control: a method for patient-cooperative robot-aided gait rehabilitation. IEEE Trans Neural Syst Rehabil Eng.18(1): 38-48. 2010.

[7] Cai LL. Fong AJ. Otoshi CK., et al.: Implications of assist -as-needed robotic step training after a complete spinal cord injury on intrinsic strategies of motor learning. J Neurosci 26:10564-10568. 2006.

[8] J Mehrholz, S Thomas, C Werner, J Kugler, M Pohl, B Elsner. Electromechanical -assisted training for walking after stroke. Cochrane Database Syst Rev. 5(5): CD006185, 2017.

[9] J Perry and J M. Burnfield. Gait Analysis: Normal and Pathological Function. Second edition. Slack Inc., 2010. 